\title{
SOME REMARKS ON COUNTABLY BARRELLED AND COUNTABLY QUASIBARRELLED SPACES
}

\author{
by SUNDAY O. IYAHEN
}

(Received 5th January 1967)

Barrelled and quasibarrelled spaces form important classes of locally convex spaces. In (2), Husain considered a number of less restrictive notions, including infinitely barrelled spaces (these are the same as barrelled spaces), countably barrelled spaces and countably quasibarrelled spaces. A separated locally convex space $E$ with dual $E^{\prime}$ is called countably barrelled (countably quasibarrelled) if every weakly bounded (strongly bounded) subset of $E^{\prime}$ which is the countable union of equicontinuous subsets of $E^{\prime}$ is itself equicontinuous. It is trivially true that every barrelled (quasibarrelled) space is countably barrelled (countably quasibarrelled) and a countably barrelled space is countably quasibarrelled. In this note we give examples which show that (i) a countably barrelled space need not be barrelled (or even quasibarrelled) and (ii) a countably quasibarrelled space need not be countably barrelled. A third example (iii) shows that the property of being countably barrelled (countably quasibarrelled) does not pass to closed linear subspaces.

(i) Let $E$ be the strong dual of a metrisable locally convex space. Then by $((1)$, pages 71 and 88$), E$ need not be quasibarrelled. But $E$ is countably barrelled, being countably quasibarrelled and complete ((2), Propositions 1 and 4).

(ii) Denote by $c$ the Banach space of all convergent sequences $x=\left(x_{1}, x_{2}, \ldots\right)$ with the supremum norm, by $c_{0}$ the closed linear subspace of $c$ consisting of sequences converging to zero and by $\phi$ the linear subspace consisting of all sequences containing only a finite number of non-zero entries. For each $n$, let $f_{n}$ be the linear functional on $\phi$ defined by the equation $f_{n}(x)=n x_{n}$. As pointed out by Weston $((4)$, page 1$),\left(f_{n}\right)$ is a pointwise bounded sequence of continuous linear functionals on $\phi$ (under the norm topology induced from $c$ ) which is not equicontinuous. Thus by Corollary 6 of (2), $\phi$ is not countably barrelled, though it is countably quasibarrelled, being bornological.

(iii) Since any separated locally convex space is a closed linear subspace of some barrelled space ((3), Theorem 1.1), to show that a closed linear subspace of a countably barrelled (countably quasibarrelled) space need not be of the same sort, it is sufficient to give an example of a separated locally convex space which is not countably quasibarrelled. Let $(E, u)$ be $c_{0}$ with the supremum 
norm $u$ and let $v$ be the associated weak topology on $c_{0}$. For each $n$, let $g_{n}$ be the linear map from $(E, v)$ into $(E, u)$ defined as follows:

$$
g_{n}(x)=\left(x_{1}, x_{2}, \ldots, x_{n}, 0,0,0, \ldots\right) .
$$

Then $\left(g_{n}\right)$ is a sequence of continuous linear maps from $(E, v)$ into $(E, u)$ such that, for each $x$ in $E, g_{n}(x)$ converges to $x$ in $(E, u)$. Moreover, $\left(g_{n}\right)$ is uniformly bounded on bounded sets, for if $B$ is the unit ball in $(E, u)$, the union over $n$ of $g_{n}(B)$ is contained in $B$. But $\left(g_{n}\right)$ is not equicontinuous since $v$ is strictly coarser than $u$. Therefore by Corollary 6 of $(2),(E, v)$ is not countably quasibarrelled.

\section{REFERENCES}

(1) A. Grothendieck, Sur les espaces $(F)$ et $(D F)$, Summa Brasil. Math. 3 (1954), 57-122.

(2) T. Husain, Two new classes of locally convex spaces, Math. Ann. 166 (1966), 289-299.

(3) Y. Komura, On linear topological spaces, Kumamoto J. Sci. Ser. A 5 (1962), 148-157.

(4) J. D. Weston, The principle of equicontinuity for topological vector spaces, Proc. Univ. Durham Philos. Soc. Ser. A 13 (1957), 1-5.

UNIVERSITY OF KEELE

ENGLAND 